\title{
A family study of hidradenitis suppurativa
}

\author{
J S FITZSIMMONS AND P R GUILBERT
}

From the Department of Clinical Genetics, City Hospital, Hucknall Road, Nottingham NG5 IPB.

SUMMARY A family study of hidradenitis suppurativa was undertaken based on 26 subjects with the disease. The probands were obtained from Hospital Activity Analysis (HAA) records for a three year period (1980 to 1983) and by direct referral from hospital specialists over a six month period (1983 to 1984). Family pedigree information was collected by home visits or hospital interviews and confirmation of the disease in relatives was obtained by examination where possible or by telephone contact and hospital/GP records. A total of 62 affected persons was eventually ascertained, 40 females and 22 males. In 11 families there was evidence in favour of a genetic aetiology with single gene transmission. In another three families there was historical evidence of familial occurrence and in nine families no family history was obtained at the time of enquiry. Problems of ascertainment, including variability of age of onset and psychosocial factors, were noted and could be responsible for false negative family histories or underestimation of affected persons.

Hidradenitis suppurativa is a chronic inflammatory condition of the skin and subcutaneous tissues in those areas containing apocrine glands. ${ }^{1}$ In the early stages the lesions begin as painful subcutaneous nodules which may rupture on to the surface of the skin or progress to formation of abscesses with cellulitis, sinus formation, and subcutaneous tunnelling. Relapses and remissions are common, but in the chronic phase there is usually marked induration and scarring. The apocrine glands are widely scattered over the body surface, but are concentrated mainly in the axillae, perianal regions, labia, scrotum, lower back, and in and around the breasts. It is these areas which are most often affected, although the disease is more common in the axillae. The age of onset varies from adolescence to early or even middle adult life and affects both sexes, but is reported to be more common in females. The condition tends to become quiescent after years of activity and although antibiotics may be of some value before induration and scarring occur, treatment in the latter stages is unsatisfactory and many patients resort to surgery. This usually consists of simple excision of the diseased area or may involve more widespread excision of the hair-bearing area with subsequent skin grafting. Despite the chronic and relapsing nature of the disease, the general health of the majority of patients remains surpris-

Received for publication 29 September 1984

Accepted for publication 8 October 1984 ingly good. A number of complications have been reported, including anaemia, ${ }^{2}$ septicaemia, ${ }^{3}$ fistula formation involving pelvic organs, ${ }^{4}$ and, in a small number of patients, neoplastic change. ${ }^{5}$ Interstitial keratitis $^{6}$ and non-specific spondyloarthropathy ${ }^{7}$ have also been described, the latter more commonly in those with associated acne conglobata.

The cause of hidradenitis suppurativa is unknown, but various precipitating factors have been described, including the use of depilating agents, deodorants, poor personal hygiene, and constricting clothing. Acute lesions of the apocrine glands can be produced experimentally by the application of occlusive tape to the axillae. ${ }^{8}$ Obesity and diabetes have been noted in occasional patients, but there is no evidence of a specific endocrine factor in the aetiology of this disease. Hidradenitis suppurativa has been classified with acne conglobata and dissecting cellulitis of the scalp as the follicular occlusive triad. ${ }^{9}$ Although all three may occur singly or together, acne conglobata or cystic acne is thought to be the most common lesion of this group and dissecting cellulitis of the scalp is rare. The primary cause of the poral occlusion in these disorders remains obscure, but the presence of comedones due to the collection of keratinous material appears to be important. Dermatologists generally accept that poral occlusion occurs initially in the pilosebaceous apparatus secondary to comedone formation at the outlet of the sebaceous gland. This is thought 
to produce the obstruction of the apocrine gland which is closely associated with the pilosebaceous systems, and the obstructed glands subsequently distend and then become infected. However, although some affected subjects develop cystic acne during adolescence, only a small number develop chronic hidradenitis suppurativa, and other factors must be important.

The chronic and recurring nature of the condition suggests the possibility of a defect in the host defence mechanisms, but investigations of a number of affected patients have failed to confirm any generalised immunological abnormality. ${ }^{10}$ Staphylo- coccus aureus, streptococci, and gram negative $\Rightarrow$ organisms have been cultured from chronic lesions, but in some cases the acute lesions remain sterile, at? least in the initial phase. It seems almost certain that infection is a secondary and not a primary event. There have been no reports of any changes in the $\mathcal{D}$ anatomical structure of the apocrine gland although this possibility was referred to in one report. ${ }^{11}$

\section{Family studies}

The probands in this study were ascertained from HAA records from a busy district general hospitali

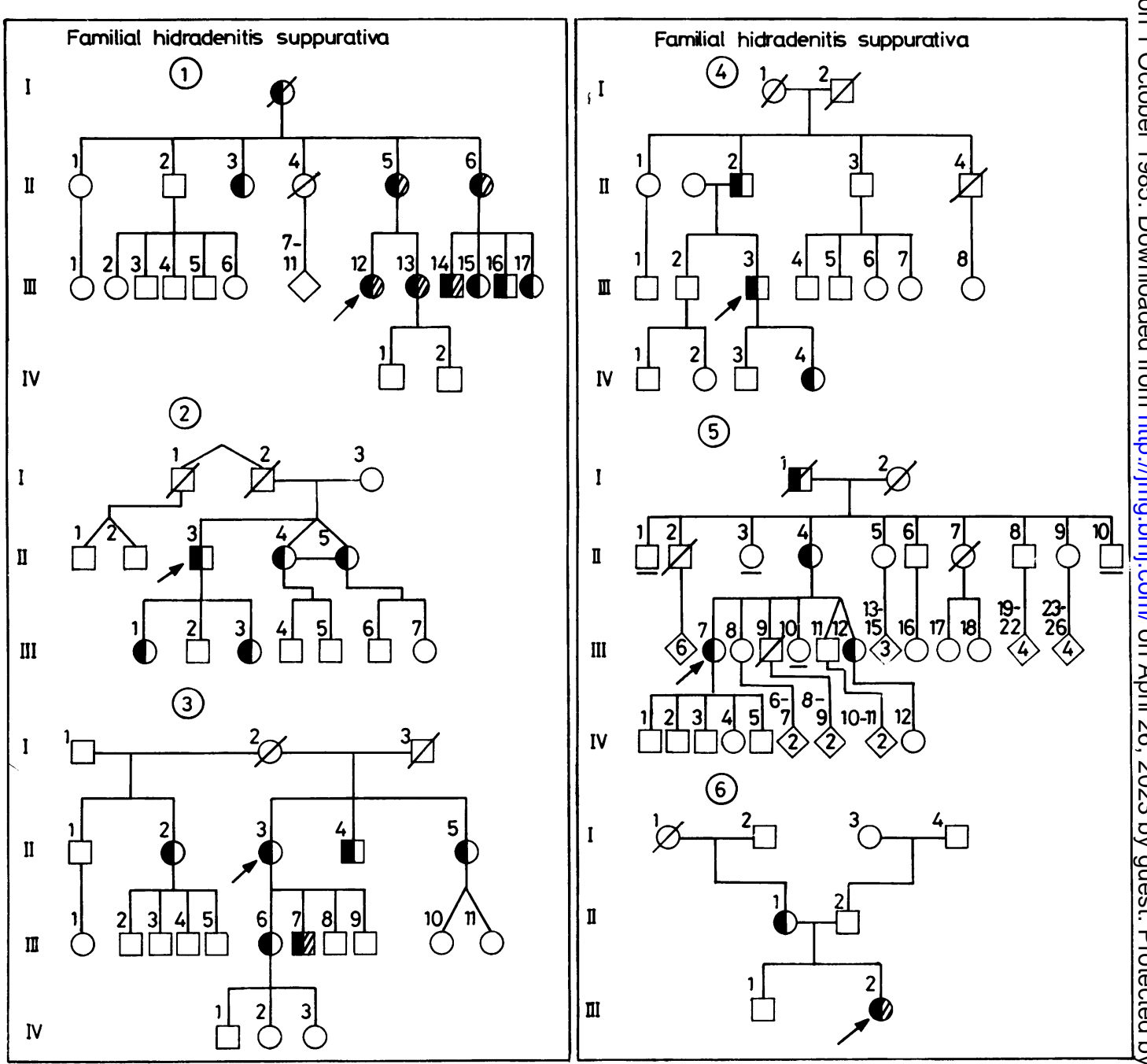




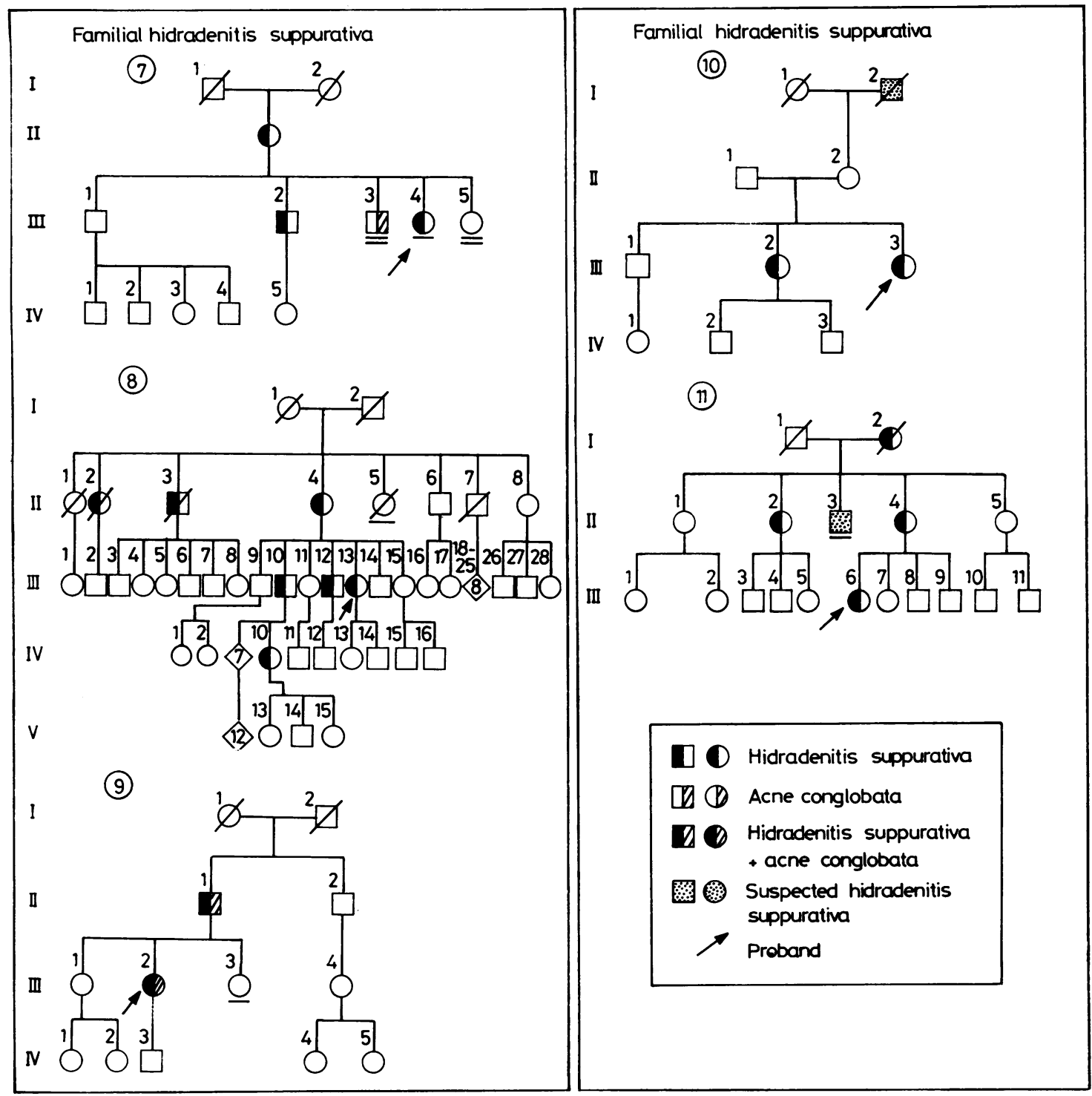

FIG 1 Pedigrees of families 1 to 11. There are 48 affected persons, eight only with associated acne conglobata. Male to male transmission in family 4.

providing specialist plastic surgical services for the East Midlands. Probands were also obtained from individual hospital specialties, including dermatology and general surgery. There was considerable overlap in patients referred from these two sources. HAA records deal only with inpatients and outpatients referred to specialties other than those mentioned would not be included in this study.
General practitioners were not contacted and ascertainment was undoubtedly incomplete. The total number of patients identified with the disease eventually totalled 62,40 female and 22 male. Forty-eight of these were from 11 families (fig 1), three from three families (fig 2), and nine from nine families (fig 3). Two probands did not agree to cooperate in further family investigations. 
? Familial hidradenitis suppurativa

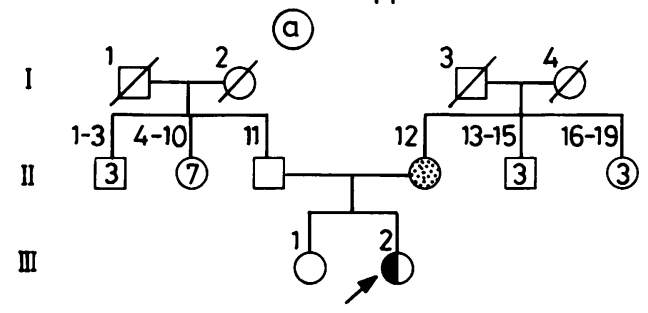

(b)
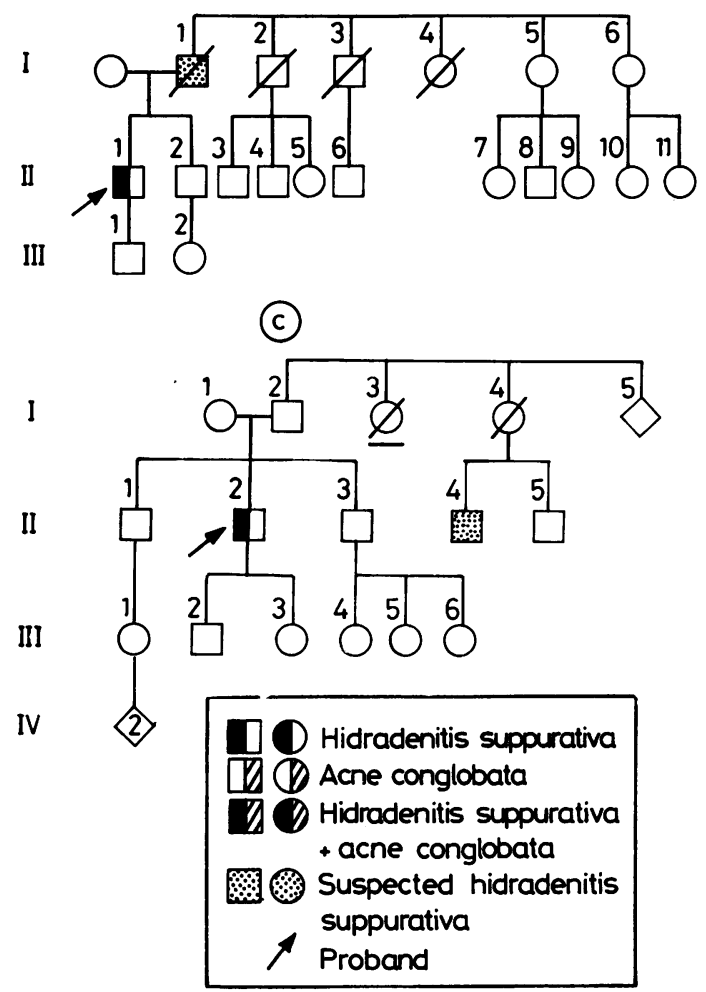

FIG 2 Pedigrees of families $a, b$, and $c$. Apparent male to male transmission in family $b$.

In the 11 families (fig 1 ) with 48 affected subjects there are 35 females and 13 males. The disease is transmitted vertically over three generations in five families and over two in six. In one family (family 4) there is male to male transmission. Identical twins II. 4 and II.5 in family 2 were both affected.

In three families (fig 2) a history of the disease was obtained in a parent of the proband in two of these families and a cousin of the proband in the third. It seems likely that further information about these subjects will be obtained. In family b, I.1 died from other causes but this pedigree is important as it demonstrates male to male transmission.

In nine families (fig 3) we have been unable to confirm the disease in any other subjects, although investigations continue. Initially, this group included a family where it was presumed there was only one affected patient. However, subsequent contact with a cooperative, knowledgeable, key member confirmed that the proband's mother and sister both suffered from hidradenitis suppurativa as did their mother in generation I. This is family 11 in fig 1 .

\section{Discussion}

Not all diseases occurring in more than one member of a family are necessarily genetic and familial clustering may be seen in many disorders, including those of environmental origin. However, the number of affected persons in the families shown in fig 1 and the absence of any other obvious cause favours a genetic aetiology. The fact that males and females are both affected and the vertical transmission of the disease through several generations is consistent with dominant inheritance. One family (family 4) definitely, and another family (family 2) probably, demonstrate male to male transmission making it more likely to be autosomal dominant than sex linked dominant. The number of affected first degree relatives, that is, parents, sibs, and children of the probands, is approximately $34 \%$ of the total number of first degree relatives of the 11 families (table). This falls short of the expected $50 \%$ but it is not inconsistent with the proposal that the disease is inherited in an autosomal dominant fashion. As with other genetic disorders, variation in the age of onset, incomplete ascertainment, and variable penetrance may distort the expected proportion of affected persons and make straightforward segregation analysis difficult. In this study, there were at least 40 first degree relatives of affected patients shown in fig 1 who were under the age of 20 at the time of investigation and could therefore theoretically develop the disease at some stage in the future. Forty-six of the total 62 affected persons developed hidradenitis in their late teens or early adult life and 16 of the total between the ages of 30 and 40 . Another factor of importance in accurate ascertainment was the reluctance of some females to admit to the disease even to close relatives and, in some cases, to their parents. Chronic vulval and perianal lesions were a source of embarrassment to many affected women and this proved a difficult problem throughout the study. In addition, there was evidence of intrafamilial tension in a number of families investigated and this was presumed to be a 
A family study of hidradenitis suppurative

371

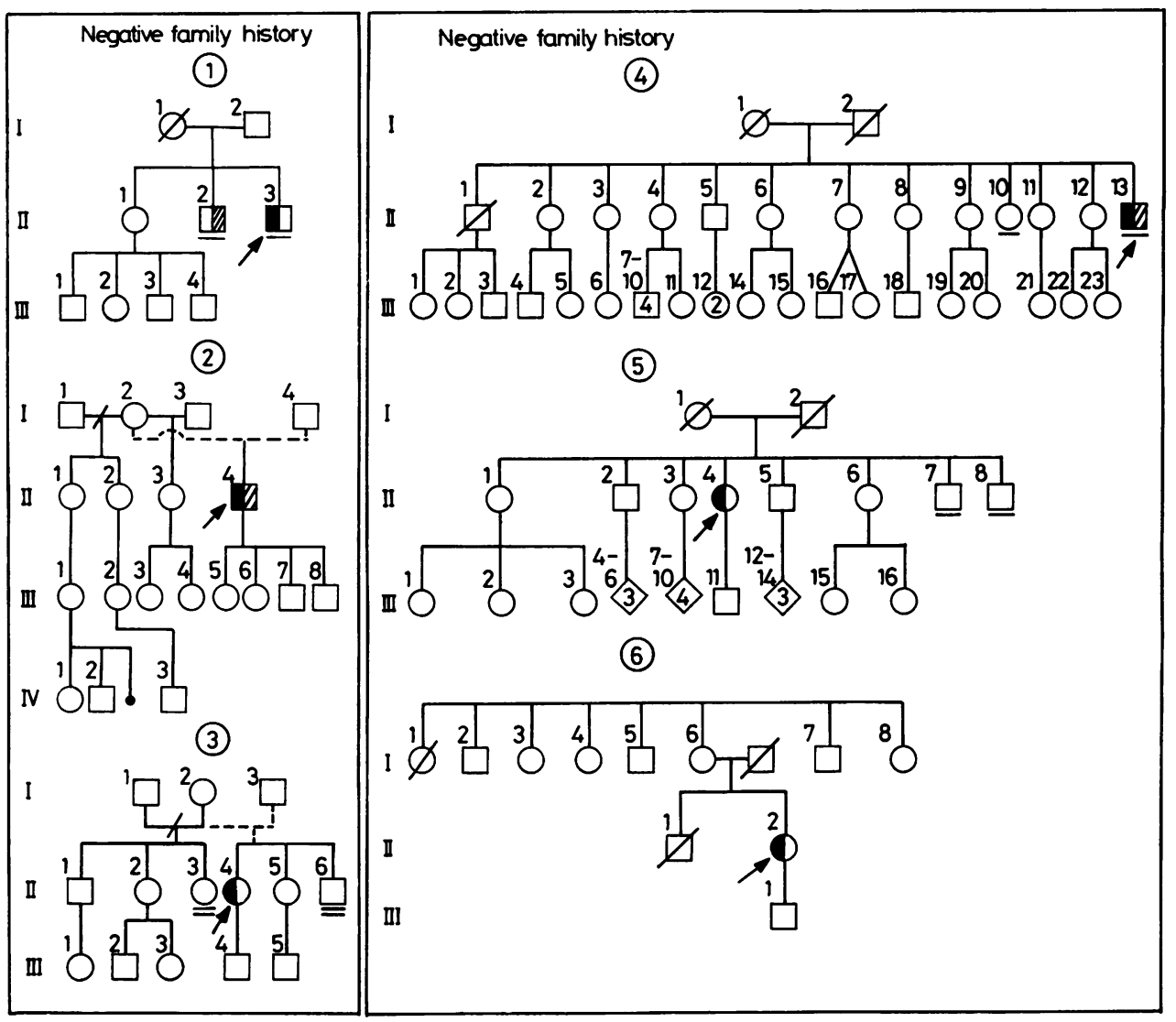

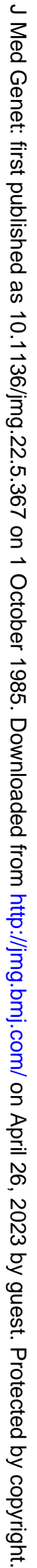

FIG 3 Pedigrees of families 1 to 9. At the time of ascertainment there was a single affected person in each family. 
TABLE Frequency of hidradenitis suppurativa in first degree relatives of probands.

\begin{tabular}{lllll}
\hline Relationship & Total & Number affected & $\%$ affected & Expected \\
\hline Sibs & 29 & 10 & $\approx 35$ & \\
Parents & 22 & 8 & $\approx 36$ & $50 \%$ \\
Children & 19 & 6 & $\approx 31$ & \\
Total & 70 & 24 & $34 \cdot 3$ & \\
\hline
\end{tabular}

consequence of the implied hereditary nature of the disease and its emotional affects. This is particularly well exemplified by family 2 in fig 1 . The proband (II.3) was completely unaware that either of his two daughters III.1 or III. 3 had the disease. When III.1 was first contacted, she denied knowledge of the condition but subsequently telephoned to admit that she was a sufferer but was too embarrassed to let it be known to her father or other family members. Her sister, who was also affected, was very relieved to discuss the problem which she had had for many years. Other factors likely to influence ascertainment are variation in the severity of the disease and the fact that the condition tends to burn itself out after the menopause. Some older patients could forget about the condition and this was noted on several occasions. Although clinical variation obviously does occur, the majority of affected persons have a definite history of many episodes of infection over the years, and most consider the condition to be an extremely burdensome and distressing one.

It is difficult to explain the absence of the disease in other sibs and first degree relatives of the subjects in families 1 to 9 in fig 3 . The family pedigrees are strikingly different from those shown in fig 1 , but there are nine offspring of probands who were under the age of 20 years at the time of investigation and they could theoretically also develop the disease in future. It is possible that there may be aetiological heterogeneity in this disease and it could be due to genetic factors in some families but not in others. Multifactorial inheritance may, on occasions, mimic mendelism but the number of affected subjects in the families shown in fig 1 exceed that expected from a multifactorial model. There is no accurate information on the prevalence of this disease. Figures from one busy dermatological outpatient practice* suggest an incidence of approximately 1 in 3000 . If this is a reasonably accurate estimate, then the recurrence rate anticipated in first degree relatives would be approximately 1 in 50 on a

*The Department of Dermatology under the direction of Professor J A A Hunter, The Royal Infirmary, Edinburgh, provided information relevant to the possible incidence of hidradenitis suppurativa. multifactorial basis (twice the square root of the disease incidence). This is considerably less than the recurrence rate in relatives in the 11 families shown in fig 1 , and at present greater than the occurrence rate in first degree relatives in the nine families shown in fig 3 .

There was no evidence of other likely causative factors and none of the families demonstrated any consistent abnormality of social or personal habits. Detailed investigation of family 1 in fig 1 did not reveal any biochemical or chromosomal marker and clinical examination of many other affected persons merely confirmed the presence of hidradenitis suppurativa and no other associated lesions. Only eight of the total affected persons had associated acne conglobata and five of these were from family 1 in fig 1. This was an unusual family. The acne conglobata was severe and presented in some affected persons at the age of 6 to 7 years. There was no historical or clinical evidence of an increased incidence of severe acne vulgaris in the remaining affected patients and no evidence of an underlying endocrine or metabolic disturbance.

\section{Conclusion}

Most published reports on hidradenitis suppurativa have been concerned with a description of the clinical findings and treatment. There has been little or no attention paid to family details. Knaysi et $_{\text {al }} \mathrm{l}^{12}$ noted a family history in three of 18 patients specifically questioned, but gave no further information. An earlier report ${ }^{13}$ on the possibility of genetic factors in hidradenitis suppurativa concerned the first three families in this paper. Although the familial nature of hidradenitis is occasionally mentioned in older reports, pedigree information is not supplied and detailed family studies appear not to have been undertaken. Cockayne ${ }^{14}$ suggested that acne vulgaris behaved as a simple dominant, but this view was refuted by Stokes and King ${ }^{15}$ who considered the heritable factor to be complex. However, it has never been suggested that hidradenitis suppurativa is related to acne vulgaris, although it does appear to be occasionally associated with acne conglobata, as has been noted previously. The ease with which the probands and affected subjects were ascertained in this investigation would suggest that the condition is probably much commoner than suspected and further studies are necessary to establish an accurate prevalence rate in our community. If a single gene defect is the basic cause of the condition in some patients, this should be confirmed in other families. The nature of the genetic defect will need further investigation. Detailed morphological studies of the apocrine glands 
in affected and at risk subjects may be rewarding, but would have to be done early in the disease before the structure of the glands was too severely disrupted. An anatomical abnormality of these glands has been commented on, but no confirmatory evidence has been offered. The symmetrical nature of the condition is striking in most cases and is difficult to explain. Anticipated further developments in molecular genetics make it important to look for abnormalities of keratin or other tissues which may become amenable to gene probing techniques. In addition, there is a need for more detailed clinical information about affected subjects, particularly the time of onset, the presence of acneiform lesions, and the natural history of the disease.

The authors wish to thank the consultant dermatologists, plastic surgeons, and other clinicians who kindly referred patients for inclusion in this study. We would also like to thank Dr G Corney, The Galton Laboratory, University College London, and Dr K Buckton, MRC Clinical and Population Cytogenetics Unit, Western General Hospital, Edinburgh, for their help in excluding known biochemical or cytogenetic markers in family 1 in fig 1 . We are grateful to Miss P C Webster who typed the manuscript.

\section{References}

${ }^{1}$ Brunsting HA. Hidradenitis and other variants of acne. Arch Dermatol 1952;65:303-15.
2 Gordon SW. Hidradenitis suppurativa: a closer look. J Natl Med Assoc 1978;70:339-43.

${ }^{3}$ Moschella SL. Hidradenitis suppurativa: complications resulting in death. JAMA 1966;198:83-5.

${ }^{4}$ Ching CC, Stahlgren LH. Clinical review of hidradenitis suppurativa. Management of cases with severe perianal involvement. Dis Colon Rectum 1965;8:349-52.

${ }^{5}$ Humphrey LJ, Playforth H, Leavell UW, et al. Squamous cell carcinoma arising in hidradenitis suppurativum. Arch Dermatol 1969;100:59-62.

${ }^{6}$ Rosner IA, Richter DE, Huettner TL. Spondyloarthropathy association with hidradenitis suppurativa and acne conglobata. Ann Intern Med 1982;97:520-5.

7 Bergeron JR, Stone OJ. Interstitial keratitis associated with hidradenitis suppurativa. Arch Dermatol 1967;95:473-5.

${ }^{8}$ Brunsting HA. Hidradenitis suppurativa: abscess of the apocrine sweat glands. Arch Dermatol Syph 1939;39:108-20.

9 Self SJ, Montes LFL. Follicular occlusion triad. South Med J 1970;63:156-60.

${ }^{10}$ Dvorak VC, Root RK, MacGregor RR. Host-defense mechanisms in hidradenitis suppurativa. Arch Dermatol 1977;113:450-3.

11 Sulzberger MD, Baer RL. Treatment of pyodermas (common pus-forming infections of the skin). In: The 1950 yearbook of dermatology and syphilology. Chicago: Yearbook Publishers, 1951:46.

12 Knaysi GA, Cosman B, Crikelair GF. Hidradenitis suppurativa. JAMA 1968;203:19-22.

13 Fitzsimmons JS, Fitzsimmons EM, Gilbert G. Familial hidradenitis suppurativa: evidence in favour of single gene transmission. J Med Genet 1984;21:281-5.

14 Cockayne EA. Inherited abnormalities of the skin and its appendages. London: Oxford University Press, 1933:351.

is Stokes JH, King AD. Acne vulgaris: heredity in the etiologic background. Arch Dermatol Syph 1932;26:456.

Correspondence and requests for reprints to $\mathrm{Dr} \mathrm{J} \mathrm{S}$ Fitzsimmons, Department of Clincial Genetics, City Hospital, Hucknall Road, Nottingham NG5 1PB. 